\title{
Human Face (Sketch/Photo) Age Group Estimation and Classification Using Perfect Face Ratios and Levenshtein Distance
}

\author{
Khalid Ounachad ${ }^{1}$, Mohamed Oualla ${ }^{2}$, Abdelalim Sadiq ${ }^{3}$ \\ ${ }^{1,3}$ Department of Informatics, Faculty of sciences, Ibn Tofail University, Kenitra, Morocco, \\ khalid.ounachad@uit.ac.ma a.sadiq@uit.ac.ma \\ ${ }^{2}$ SEISE: Software Engineering \& Information Systems Engineering Team, Faculty of sciences \& technology, \\ Moulay Ismail University, Errachidia, Morocco, mohamedoualla76@gmail.com
}

\begin{abstract}
The main objective of this paper is to identify the age group of the human being based on their face images by using a geometric feature and Levenshtein distance. This paper presents a novel method for human face age group estimation and classification. It is inspired in our model which was pre-trained on face sketch gender classification and recognition task. Toward this end, we generate some referential faces suitable for each age group decades based on perfect face ratios and four classical averages. sixteen features will be extract from the input face image based on perfect face ratio rules. The detection stage passes by Viola and Jones algorithm. The classification task is evaluated through calculate of the Levenshtein distance between the input face image request and the referential faces. An experimental evaluation demonstrates the satisfactory performance of our approach on FG-NET database with $80.00 \%$ for training, $20.00 \%$ for the testing and divided by age groups decades. Also, the CUHK Face Sketch dataset (CUFS) is used for testing. The proposed algorithm will be a competitor of the proposed relative the stat of the art approaches. The estimate rate reaches more than $79.90 \%$ for some age groups decade.
\end{abstract}

Key words: Age Group Classification, Average Face Ratios, CUFS Dataset, Face sketch, Facial Age Estimation, FGNET Database, Perfect Face Ratios, Levenshtein Distance.

\section{INTRODUCTION}

Age estimation is defined as the determination of the person's age or his/her age group [1]. The main objective of age (group age) estimation is that estimated age is as close to appearance age as possible. In the literature, usually all used algorithms take one of two approaches: age group classification or age-specific estimation [2], these approaches can be further decomposed into two decisive steps: feature extraction and classification step. Also, in both them the estimated age (group age) is the age defined automatically by computer based on persons appearance [3].

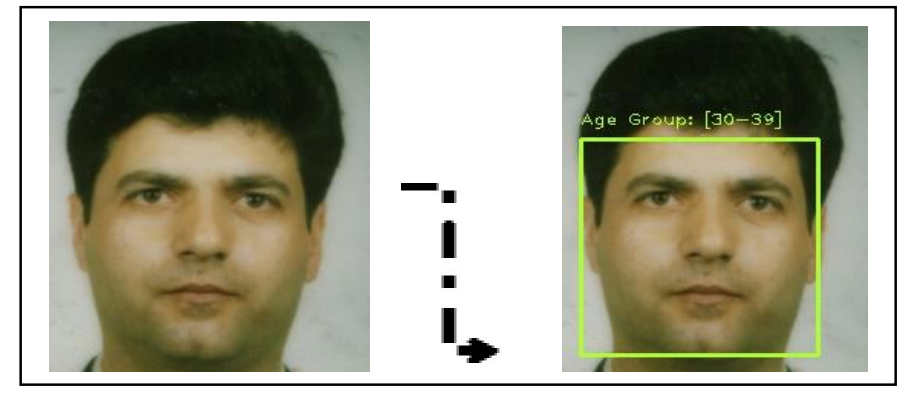

Figure 1: In the left, an input image photo to the Facial age group estimation and classification system. In the right, the output result. It indicates the detected age group.

Face Age Estimation Systems (FAES) aim to estimate age or group age in a dataset of photos or sketches images (Figure.1). It based on two-dimensional images of human subjects. Facial age estimation (FAE) is widely applicable and has great potential in many significant real-world application areas primarily in the fields of computer vision and artificial intelligence [4][5][6]. There are various application areas for age estimation including Age simulation [7], Electronic customer relationship management (chat rooms websites, forums, and emails) [8], Security and surveillance for preventing underage from accessing for preventing underage from accessing to alcohol and cigarette vending machines [9], Missing persons [10] and soon. We use age estimation and classification in our face sketch recognition system [11] and in face emotion classification [12], in this scenario it can be used to identify; fastly; a criminal person from his sketch for purposes of identification.

They are many basics kinds of age groups but the famous are those they divided age by decades ([0-9], [10-19], ... and soon), for example, in the national ID card case.

People's face is one of the most nonverbal exposed part of body. The human face adequately conveys much information, which people have a natural behavior and a remarkable ability to extract, analyze, identify, and interpret. Age is one of the most important attributes of face. 
Research ware demonstrated that age information are encrypted in the face features. The automatic task of age recognition is a challenging work and explicitly difficult: Human age estimation and classification can be done in many ways, but this paper is concerned with the age estimation and classification based on two-dimensional images of people's faces. There is a large number of databases available for human age estimation research, some of them are private and some are public. The Fg-net database [13] is most commonly used in age estimation scenario.

The Levenshtein distance [14] is a string metric for measuring the difference between two sequences. Informally, the Levenshtein distance between two words is the minimum number of single-character edits (insertions, deletions or substitutions) required to change one word into the other. It's may also be referred to as edit distance. This distance is used in this work to determine the similarity between the two images: The input request image and the age group reference image. It ensures great performances in terms of speed and accuracy.

The objective of this paper is to propose our approach based on Levenshtein distance with four generated average face ratio images to estimate and classify the age group of the input request facial image, photo or sketch. This paper presents a novel method for human age group estimation and classification. We generate four referential virtual faces suitable for each kind of facial age group based on the perfect face ratios, the decades and some classical means: arithmetic average, harmonic average, contra harmonic average and quadratic average. The principle and the core of the approach include two basic aspect. The first one grants to extract perfect face ratios for the input face photo or sketch and also to extract the same feature for each referential face and the second one allows to calculate the distance between them by using the Levenshtein distance. We use the face point landmarks to extract perfect face ratios, then sixteen features will be extract. To compute distances between each image in a set of our referential faces group age and the input facial image. The Levenshtein distance based on the similarity measure function is used.

This article is organized as follows. Section II related works. Section III background information about perfect face ratios, Levenshtein distance and four classical averages, their definitions, expressions and algorithms. In section IV, the proposed framework architecture of our approach is explained in depth. Experimental results are given in section $\mathrm{V}$. A conclusion and future work are presented in section IV.

\section{RELATED WORK}

Over the last decade until March 2020, there has been a wealth of research in Human age estimation and classification based on their facial images. specially in last two years (from 2018 to March 2020) many techniques were used in this task, for example just to name a few, we briefly review related methods for age estimation and classification: In [15] authors create an intelligent system for human age estimation by using hybrid machine learning technique. The authors of [16] use a Spatially-Indexed Attention Model (SIAM) for age estimation. in [17] the authors propose an order learning technique and they study its application to age estimation. In [18] adopt a face image age estimation based on data augmentation and lightweight convolutional neural network. In [19] the authors use a beep belief network in their facial human age estimation. In [20] the authors use a specific domain transfer learning in age estimation. In [21] authors use AgeGuess database, it is an open online resource on chronological and perceived ages of people aged 5-100.

Al-Tuwaijar et al [15] use LDA algorithm as features extraction grouped into three age classes, they use also firefly and Harmony Search algorithms in feature optimization phase and J48 algorithm in features classification. They apply the system to FG-NET dataset. Pei and Dibeklioglu [16] use end-to end architecture for age estimation from facial expression videos, called Spatially-Indexed Attention Model (SIAM). The model employs convolutional networks to learn the effective appearance features. They apply their technique to UVA-NEMO SMILE database with ages from 8 to 76 years. Lim et al [17] develop an order learning a pairwise comparator to determine the order graph of classes: 'greater than,' 'similar to,' or 'smaller than', representing ranks or priorities. They apply their facial age estimator, the proposed algorithm (supervised 6CH) to MORPH II dataset, FG-NET dataset and IMDB-WIKI dataset. In face image age estimation based on a lightweight convolutional neural network, Liu et al [18] propose an improved Shu $\square$ e Net V2 network based on the mixed attention mechanism by merging classification and regression age estimation methods. They test the technique on IMDB-WIKI and FG-NET datasets. Anjali et al [19] use active appearance and scattering transform feature extraction method which allows extract geometric and texture features, in the classification step of their facial based human age estimation they use also the deep belief network classification model. To estimate the real facial age. Jones et al [20] present AgeGuess, a simple on-line database (game) in which you can post your photos, have other people guess your age, as well as guess the age of other users. This database is an open online resource on chronological and perceived ages of people aged between 5-100.

In [21] we present a model which was pre-trained on face sketch gender classification and recognition task. The model is based on fuzzy hamming distance with geometric relationships called face ratios. We attempt to tune the same model for age group estimation and classification task and we attempt to evaluate the approach to obtain a satisfied model on adience datasets.

\subsection{Contribution of this Work}

The contribution of this work can be summarized as:

- Comprehensive performance evaluation of off line based facial age group estimation and classification by using perfect face ratios and Levenshtein distance. 
Khalid Ounachad et al., International Journal of Emerging Trends in Engineering Research, 8(7), July 2020, 3191 - 3201

- The development and evaluation of a geometric learning based human face age group classification model. with $\mathrm{N}=1002$ images from FG-NET dataset, $80 \%$ for training and $20 \%$ for testing. Also, for testing, the CUFS dataset is used to evaluate the same model.

- The proposed approach can accurately classify the right kind of the age-group's face. It is inspired in our model which was pre-trained on face sketch gender classification and recognition task. This tuned method achieves our goal by producing an estimation rate reaches more than $75 \%$ especially in the FG-NET dataset. It can be comparable with the stat of the art human face age group estimators.

\section{BACKGROUND INFORMATIONS}

\subsection{Levenshtein Distance}

Levenshtein distance measures the difference between two strings. It is equal to the minimum number of characters that must be deleted, inserted or replace to go from string A to string B. We consider that each elementary operation implemented (delete, insert or replace) has a cost of 1 . We specify that each elementary operation relates to only one character. Levenshtein distance, mathematically, is the sum of these costs. The algorithm proposed below describes the calculation of the Levenshtein distance between two strings Str1 and Str2

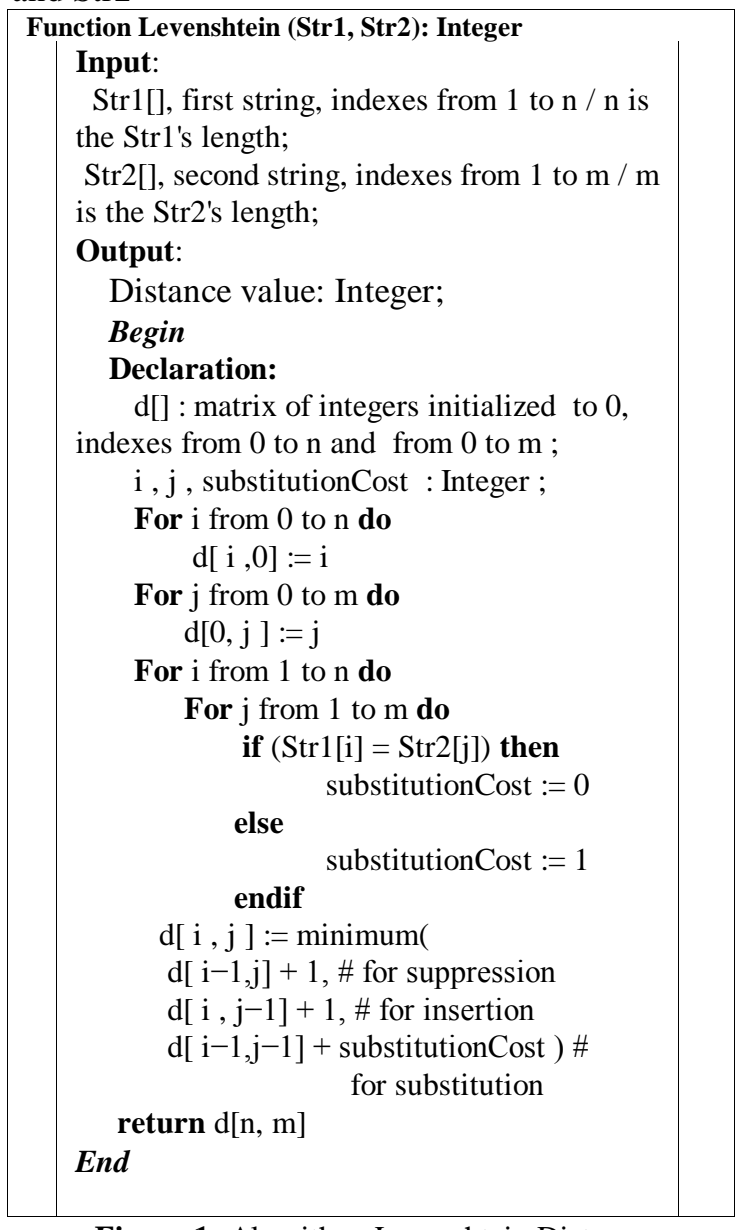

Figure 1: Algorithm: Levenshtein Distance

\subsection{Perfect face ratios}

Based on basic ideas of the Italian criminologist Cesare Lombroso. Also, based on THE IDEAL FACE PROPORTIONS taken as the scientific measuring guns of the beauty, we define the following distances as the different distances used to calculate the face ratios of each face images in datasets of face photos or sketches.

d0: The face width

d1: The distance between the end of the right eye and the right end of the face

d2: The length of the right eye

d3: The distance between the eyes

d4: The length of the left eye

d5: The distance between the end of the left eye and the left end of the face

d6: The distance between the centre of the pupils

d7: The mouth length

d8: The distance between eyes

d9: The nose width

d10: The mouth length

d11: The jaw length

d12: The distance between the eyes and the last point of the head

d13: The distance between the eyes and the chin

d14: The distance between the centre of the forehead and the last point of the head

d15: The distance between the centre of the forehead and the nose

d16: The distance between nose and the chin

d17: The distance between the eye and the eyebrow

d18: The length of one eye

d19: The distance between the low lip and the chin

d20: The facial length

We assume and we define sixteen ratios $\left(\mathrm{R}_{\mathrm{i}}\right)$ as:

$$
\begin{aligned}
& R_{1}=\frac{d 1}{d 0} \quad R_{2}=\frac{d 2}{d 0} \quad R_{3}=\frac{d 3}{d 0} \quad R_{4}=\frac{d 4}{d 0} \\
& R_{5}=\frac{d 5}{d 0} \quad R_{6}=\frac{d 6}{d 7} \quad R_{7}=\frac{d 8}{d 9} \quad R_{9}=\frac{d 10}{d 11} \\
& R_{q}=\frac{d 12}{d 13} \quad R_{10}=\frac{d 14}{d 20} \quad R_{11}=\frac{d 15}{d 20} \quad R_{12}=\frac{d 16}{d 20} \\
& R_{18}=\frac{d 17}{d 18} \quad R_{14}=\frac{d 19}{d 20} \quad \begin{array}{l}
R_{15}=\text { The nose } \\
\text { surfoce }
\end{array}
\end{aligned}
$$

Also, we assume that in a perfect face we have these ratios:

$$
\begin{aligned}
& R_{1}, R_{9}, R_{9}, R_{4}, R_{5}, R_{6}, R_{7}=0.2,0.1,0.2,0.5,0.5,0.33,0.33 \\
& R_{0} R_{9}, R_{10} R_{11}, R_{12}, R_{13}=0.33,1.0,1.0,0.5,0.2,0.2 \\
& R_{14} R_{15} R_{16}=0.2 \quad \text { < } 0.05, \quad 1.67
\end{aligned}
$$




\subsection{Arithmetic mean}

Symbolically, we have a data vector set containing $\mathrm{n}$ values $\mathrm{x}_{1}, \mathrm{x}_{2}, \ldots, \mathrm{x}_{\mathrm{n}}$. The arithmetic mean is equal to the fraction of the sum of all n_numerical values of a set and the number of items in this set. It is defined as A_Mean:

$$
\begin{aligned}
& \text { A_Mean }=\frac{1}{n} \sum_{i=1}^{n} x_{i}=\frac{S}{N} \\
& N=\text { The number of items being averaged. } \\
& S=\text { The sum of the numbers being averaged. }
\end{aligned}
$$

\subsection{Harmonic mean}

The harmonic mean is the reciprocal of the A_Mean of the reciprocals of the given numbers. It is defined as H_Mean:

$$
\text { H_Mean }=\frac{\mathrm{n}}{\sum_{\mathrm{i}=1}^{\mathrm{n}} \frac{1}{\mathrm{x}_{\mathrm{i}}}}
$$

\subsection{Contra Harmonic mean}

The contra harmonic mean is the fraction of A_Mean of the values squares $t$ and the values A_Mean. It is defined as C_H_Mean:

$$
\text { C_H_Mean }=\frac{\frac{x_{1}^{2}+x_{2}^{2}+\cdots+x_{n}^{2}}{n}}{\frac{x_{1}+x_{2} \cdots+x_{n}}{n}}=\frac{x_{1}^{2}+x_{2}^{2}+\cdots+x_{n}^{2}}{x_{1}+x_{2} \cdots+x_{n}}
$$

\subsection{Quadratic mean}

The quadratic mean is calculated as the A_mean square root of the given numbers. It is is defined as Q_Mean:

$$
\text { Q_Mean }=\sqrt{\frac{\mathrm{x}_{1}^{2}+\mathrm{x}_{2}^{2}+\cdots+\mathrm{x}_{\mathrm{i}}^{2}}{\mathrm{n}}}
$$

\section{APPROACH}

In detail, our system has two modes, in both them, the input facial age image (sketch or photo) and all face age photos of the dataset are converted to a Gray level, they are resized and cropped into 200x250 pixels. These dimensions are chosen: It's the proposed default choice of the datasets used and it's also the dimensions used in our related work. Facial images pass though viola johns facial detection algorithm [22]. In the offline phase, the first step of the system is to normalize and to pretrain all photos. For that they have been transformed into Gray level images and they are all cropped to $100 \times 125$ pixels. The same technique steps are thus used to the online mode. After this stage, to detect the faces of the images, we projected the viola and jones algorithm[23]. The result that follows this second step is used to locate the 68_point_landmarks [24] in each face. These 68 points will be the parameter of a geometric descriptor which allows to extract an identity of each face via the calculation of the rations of the perfect face.

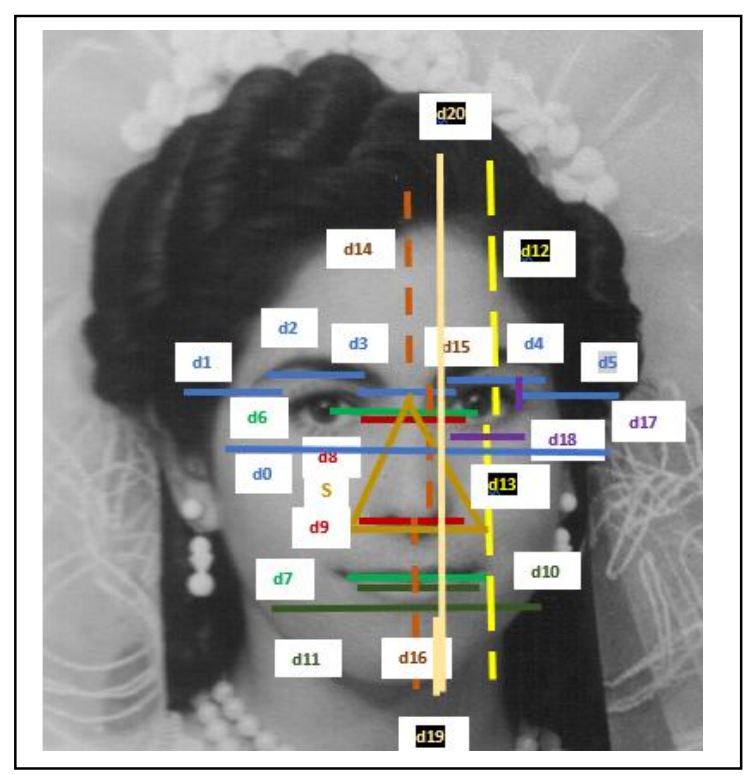

Figure 2: The different distances used to calculate the sixteen ratios of the face named 005A18 in FG-NET dataset. The ratios are inspired by cannons applied to a perfect face.

A vector will be dedicated to group these harmonious distances in order. This vector represents a real proportionality with any other similar vector. The ratios of these distances in the vector have been stored as already detailed in last section from $R_{1}$ to $R_{16}$.

An overview of our proposed Levenshtein Distance based framework for face age group classification is shown in Figure 4.

In online process of the face age group estimation and classification system, given a facial image, sixteen features are extracted based on twenty distance as showed in Figure. 3 and defined in last section. The series of these characteristics composes a vector of real values. This vector is considered as an identifier of the face image from which the values have been extracted and calculated.

In offline process of the facial age group estimation and classification System, we grouped the dataset of facial photos into seven data subsets based on the value of age group that it represents:[0-9] for the first decade,[10-19] for the second decade, [20-29] for the third decade, [30-39] the fourth decade, [40-49] fifth decade, [50-59] for the sixth decade and [60-96] for the last decade. The same distances used in online process are extracted and calculated for each facial image for each data subset in the facial age group datasets. We generate four referential faces suitable for each kind of facial age group based on the four usual and classical averages: Arithmetic mean, Contra harmonic mean, Harmonic mean and Quadratic mean. We use the landmarks points to generate all referential faces. Figure 5 summarize and shown the twenty-eight referential faces per averages per age group decade. The second column contained the seven referential faces generated by arithmetic average; they are in orange 
color. The third column contained the next seven referential faces generated by harmonic average; they are in red color. The fourth column contained the next seven referential faces generated by contra- harmonic average, they are in purple color and the last column contained the last seven referential faces generated by quadratic average, they are in blue color. After such a facial image (sketch or photo) and referential faces transformation to vectors of reals values, Facial age group estimation and classification becomes straightforward. We can compare the facial image with the referential facials age group by using the Levenshtein distance. In fact, we first compute the landmarks points for each facial image and also for all referential faces of age group. The face ratios for each one is then used as feature vectors for final estimation and classification.

The algorithm contained eight steps; the detail of algorithm can be summarized as follows.

Step-1: To pretrain and to normalize all the facial images sketches or photos.

Step-2: Extract the landmarks points of the facial images according to the famous model "The 68_face_landmarks"

Step-3: Calculate the distances $\mathrm{d} 0$ to $\mathrm{d} 20$ as defined in section III.B.

Step-4: Calculate S: the surface of the nose.

Let $\mathbf{S}$ the surface of the nose, we define the ratio:

$$
R_{15}=s
$$

Step-5: Let define:

- $\quad E^{\prime}=$ The tragus ear center
- $\quad$ I'= The inter eyebrow center

- $\mathrm{F}^{\prime}=$ The front center

- $\quad C^{\prime}=$ The chin center

- $\quad \mathrm{N}^{\prime}=$ The nose dorsum

Let define three angles $\theta 1, \theta 2$ and $\theta 3$ as:

$$
\begin{aligned}
& \theta 1=F^{\prime} E^{r} l^{\prime} \\
& \theta 2=I^{\prime} E^{\top} N^{\prime} \\
& \theta 3=N^{\prime} E^{\prime} C^{r}
\end{aligned}
$$

The following angles are calculated as:

$$
\begin{aligned}
& \theta 1=\frac{\arccos \left(E^{\prime} F^{\prime 2}+E^{\prime} I^{r 2}-F^{\prime} I^{\prime 2}\right)}{2 * E^{r} F^{r} * E^{r} I^{P}} \\
& \theta 2=\frac{\arccos \left(E^{r} N^{\prime 2}+E^{r} l^{r 2}-N^{r} I^{r 2}\right)}{2 * E^{r} N^{r} * E^{r} l^{r}} \\
& \theta 3=\frac{\arccos \left(E^{r} N^{\prime 2}+E^{r} C^{2}-N^{r} C^{2}\right)}{2 * E^{r} N^{r} * E^{r} C^{r}}
\end{aligned}
$$

We define the ratio $\mathrm{R}_{16}$ :

$R_{16}=\frac{\theta 1+\theta 2}{\theta 3}$

Then:

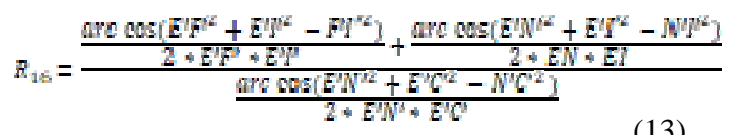

$$
\text { Step-6: Create the ratios vector: }
$$

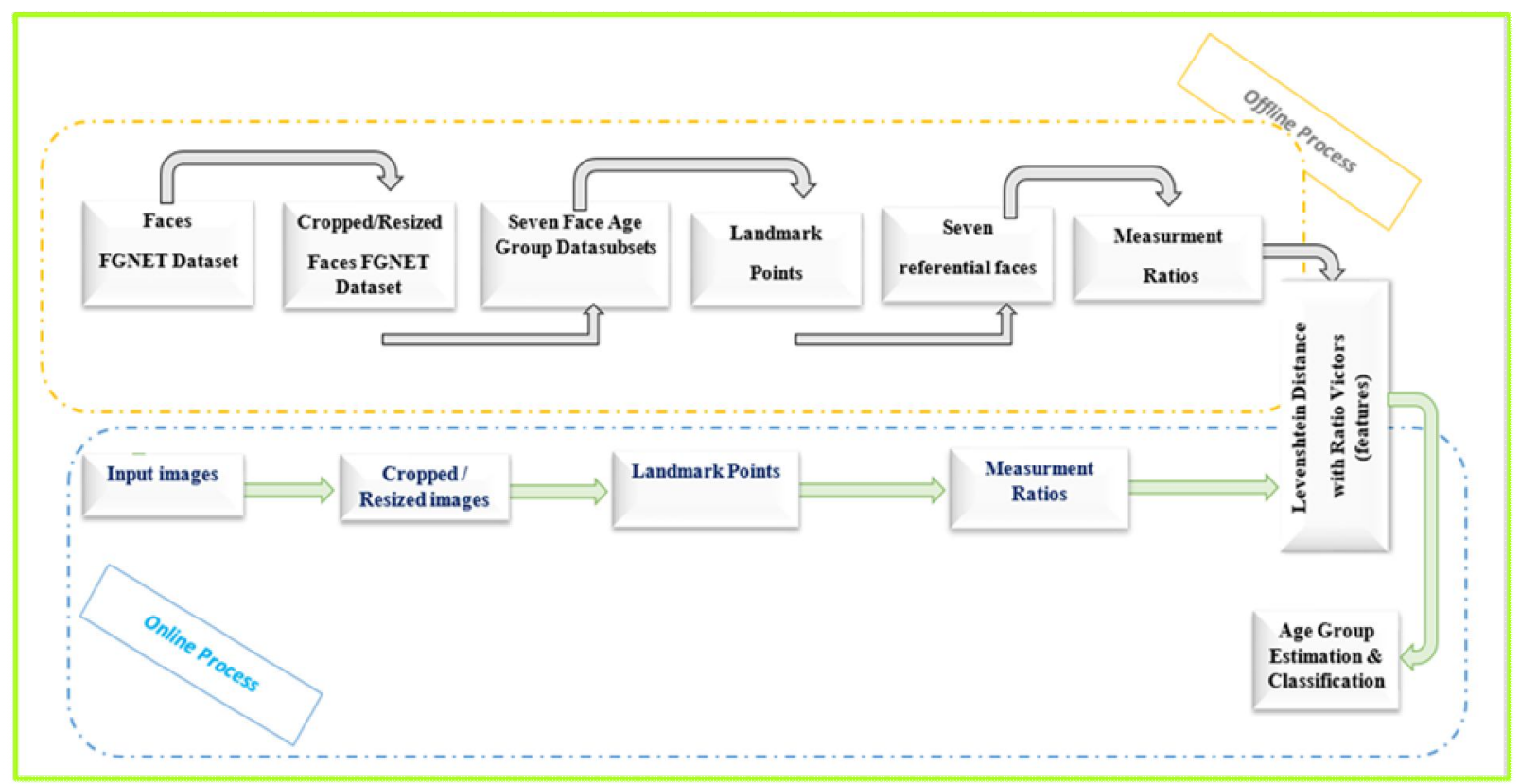

Figure 3: An overview of our proposed framework based on Levenshtein distance with face ratios for Face age group estimation and classification. 
Khalid Ounachad et al., International Journal of Emerging Trends in Engineering Research, 8(7), July 2020, 3191 - 3201

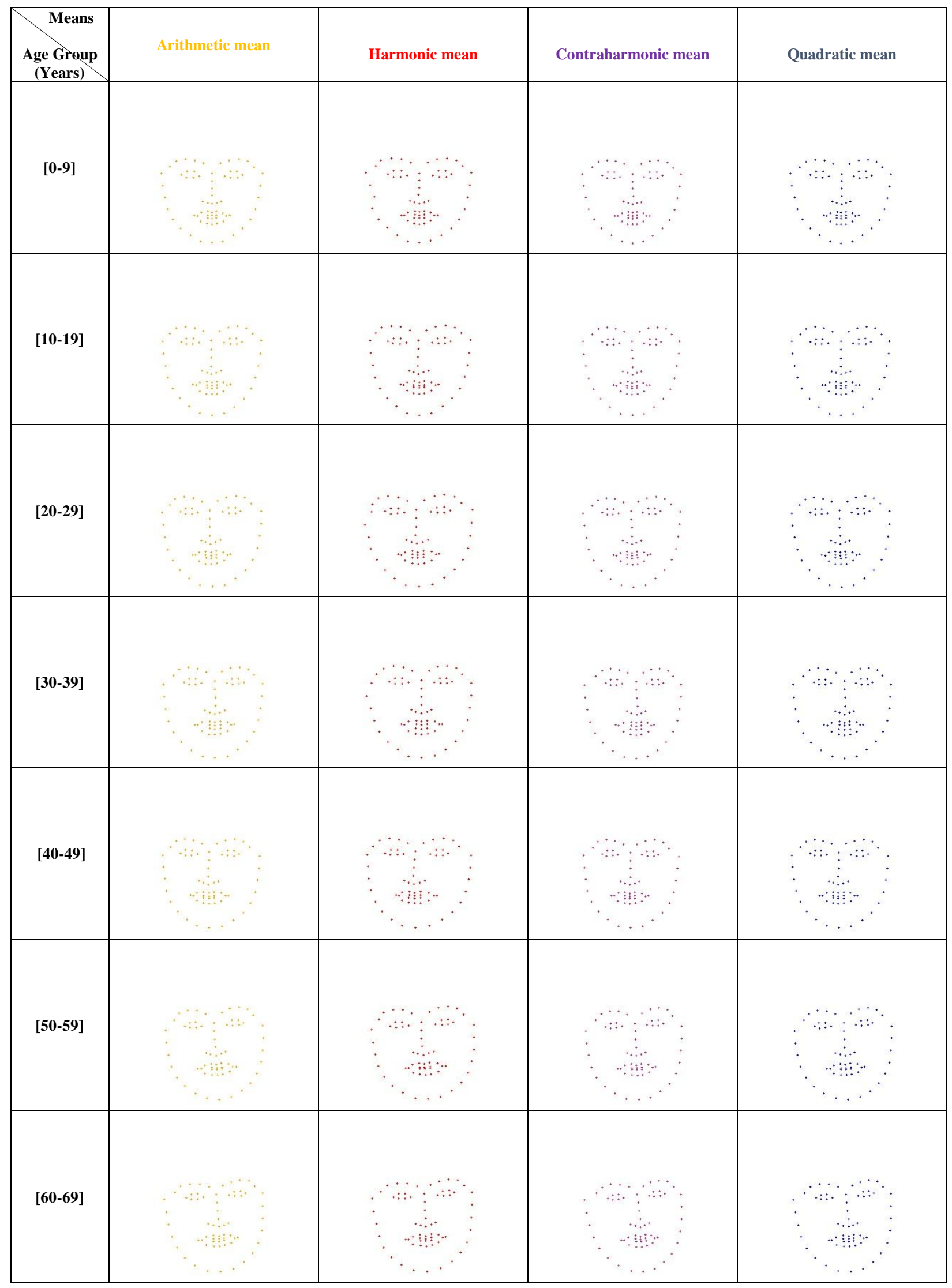

Figure 4: Our generated referentiels 68_face age group landmarks attributed to their age group interval and the name of mean used to calculate its, each model contained: "Mouth", "Right_Eyebrow", "Left_Eyebrow", "Right_Eye","Left_Eye", "Nose" and "Jaw". The images for the usual means are 200x250 like the input image request resolution. 
Step-7: Compute the Levenshtein distance (LevD) between the input facial vector $(\mathrm{Vi})$ and each $(\mathrm{Vf})$ vector in the list of referential face age group vectors.

Step-8: Estimation and classification: The output result is the age group label of the min of Levenshtein distances relatively to each input parameter.

\section{EXPERIMENTS AND RESULTS}

There is a large number of databases available for human age estimation research, some of them are public and some are private. The FG-NET database [13] is public and most commonly used in age estimation research.

To demonstrate the e $\square$ ectiveness of the proposed method, we proceeded for acquiring the FG-Net Aging Data. We used $80 \%$ of database images for training and $20 \%$ for testing. Also, for testing, the CUFS dataset is used to evaluate the same model. FG-NET includes 1002 face photos in total. In Figure.6 the FG-NET age distribution by year is detailed.

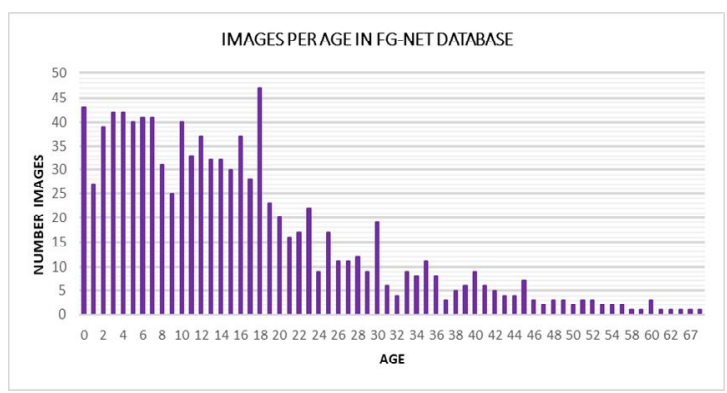

Figure 5: FG-NET age distribution

We divide the FG-Net dataset to seven data subsets suitable for each kind of basics decades: [0-9] years, [10-19] years .... and soon. Table 1 shown the percentage of each decade age group. In Figure.7 our used FG-Net Age Group statistics is detailed whilst the Figure.7 the distribution of age group used in our approach is summarized.
Table 1: The statistics of our used age groups from FGNET dataset.

\begin{tabular}{|c|c|c|c|c|}
\hline \multirow{2}{*}{$\begin{array}{c}\text { Age } \\
\text { Group } \\
\text { (Years) }\end{array}$} & \multicolumn{3}{|c|}{ Number images } & \multirow{2}{*}{$\begin{array}{c}\text { Total } \\
\text { Percentage }\end{array}$} \\
\cline { 2 - 4 } & Train & Test & Total & \\
\hline$[0-9]$ & 297 & 74 & 371 & $37.03 \%$ \\
\hline$[10-19]$ & 271 & 68 & 339 & $33.83 \%$ \\
\hline$[20-29]$ & 115 & 29 & 144 & $14.37 \%$ \\
\hline$[30-39]$ & 63 & 16 & 79 & $07.88 \%$ \\
\hline$[40-49]$ & 36 & 10 & 46 & $04.59 \%$ \\
\hline$[50-59]$ & 12 & 03 & 15 & $01.50 \%$ \\
\hline$[60-69]$ & 07 & 01 & 08 & $00.80 \%$ \\
\hline
\end{tabular}

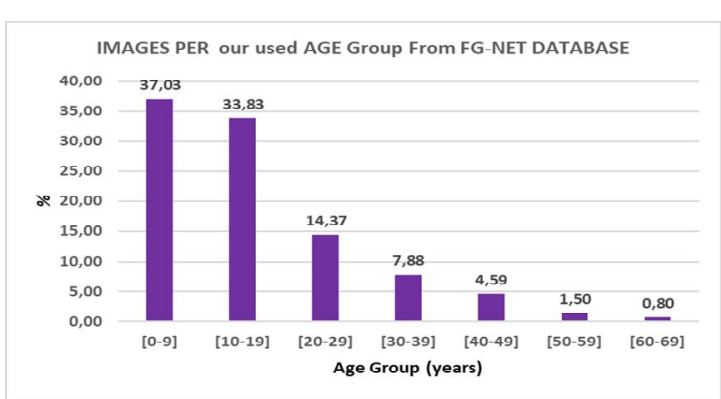

Figure 6:Our used FG-Net Age Group distribution

The FG-NET is used to training and to testing our approach. Figure 8 clearly illustrates step by step the results obtained as we progress in the process of the framework already described previously.

For each age group data subset, in the first step the extract face photos of the data subset. In the second: The cropped Face photos or sketches. In the third: The extract 68 face landmarks points. In the fourth: calculate of the sixteen features and in the last step: the ratios vector generated from the facial images, it is involved to classify the kind of the facial age group of the input face photo or facial sketch. The output result of Face Age Estimation System (FAES) is the probe kind of age group by years.

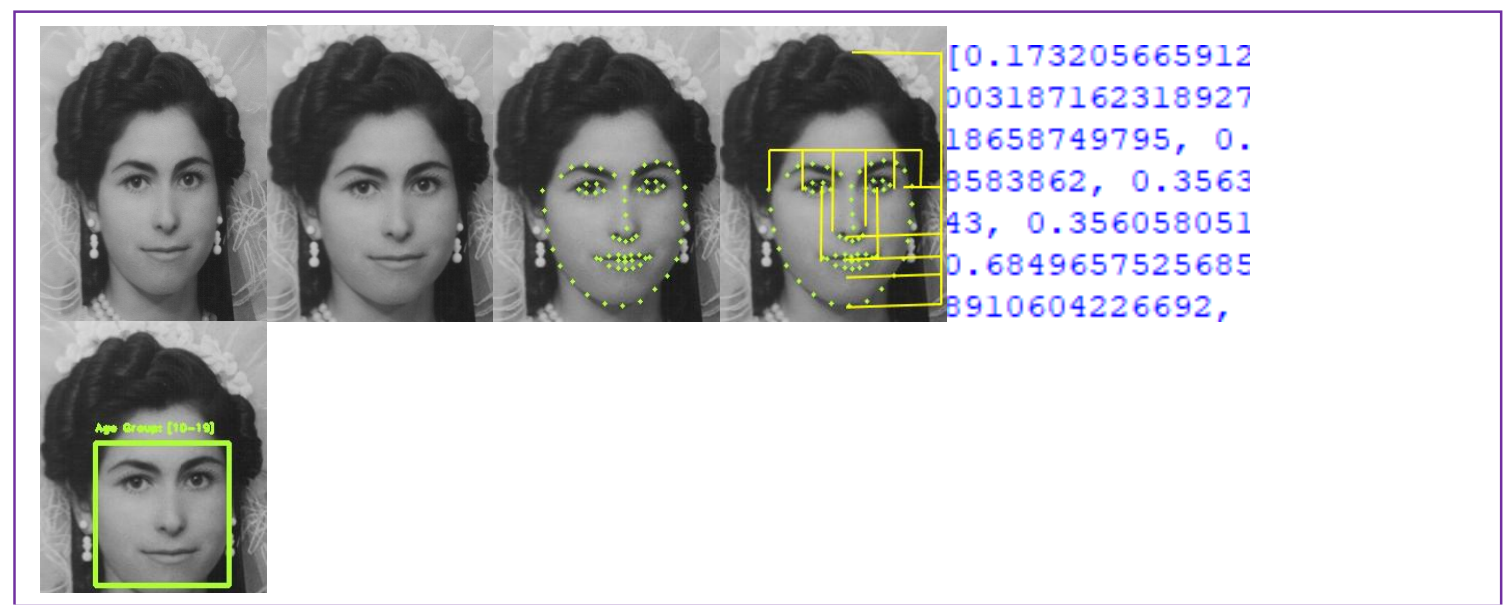

Figure 7:The process of our FEAS, line1: extract of the dataset photos/input facial images. Line2: cropped images. line3: extract the 68 face landmarks points. Line 4: calculate of sixteen features and line5: the ratio vector generated from the facial photos/sketches; it is involved to estimate the kind of age group. The output result of FEAS is the probe kind of age group of the input facial images. 
We generate seven referential faces suitable for each age group decade based on perfect face ratios and four classical averages: arithmetic mean, harmonic mean, contra-harmonic mean and quadratic mean. The results are 28 referential faces type of average as already described previously in section IV. Using our new method, we realized four experiments according to the average used during the generation of the referential face. In each one of them, we compare the input facial image (features) with the seven referential faces, for the comparison we use the Levenshtein distance. The output probe kind of age group is that proper to the referential face having the minimal Levenshtein distance value. TABLE II shows the Accuracy per Age Group for our approach based on Levenshtein Distance (LevD) and all Averages Face Ratios. The result can be considered as a benchmark for the facial age group system to compare. All experimental results of tests are shown in Figures 9 to12. The cumulative match score is used to evaluate the performance of the four algorithms used. It measures the percentage of the probe age group.

TABLE II reports the facial age group estimation and classification accuracies using five different methods: arithmetic mean, geometric mean, harmonic mean, contra harmonic mean and quadratic mean and Levenshtein distance applied to FG-NET Database. Our algorithm proves that, the classification, rate reaches more than $77.80 \%$ for [0-9] age group ,more than $75.30 \%$ for [10-19] range, more than $75.40 \%$ for the third decade, more than $79.90 \%$ for [30-39] age group, more than $70.60 \%$ for [40-49] range, also, more than than $70.60 \%$ for [50-59] range and more than $76.40 \%$ for the last decade.

Figures 9 to 12 show comparison of cumulative match scores between our various facial images age group estimation and classification methods using four classical averages.

Table 2:Accuracy (\%) per Age Group per Averages (FG-NET Database)

\begin{tabular}{|c|c|c|c|c|}
\hline $\begin{array}{l}\text { Averages } \\
\text { Age } \\
\text { Group } \\
\text { (Years) }\end{array}$ & 窇 & 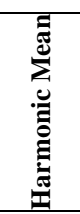 & 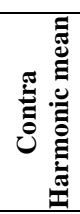 & 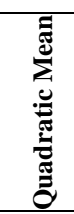 \\
\hline $\begin{array}{l}0-9] \\
\end{array}$ & 76.44 & 63.23 & 77.86 & 77.86 \\
\hline [10-19] & 59.47 & 51.22 & 51.22 & 75.38 \\
\hline [20-29] & 71.46 & 75.41 & 75.41 & 75.41 \\
\hline [30-39] & 79.95 & 58.23 & 48.01 & 48.76 \\
\hline$[40-49]$ & 61.78 & 70.67 & 65.11 & 46.22 \\
\hline [50-59] & 54.00 & 68.00 & 70.67 & $\begin{array}{l}73.33 \\
\end{array}$ \\
\hline [60-69] & 59.00 & 50.21 & 56.70 & 76.50 \\
\hline
\end{tabular}

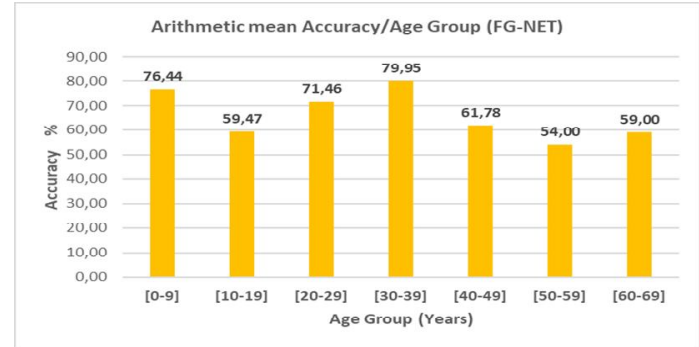

Figure 8: Performance of Arithmetic mean approach vs Age Group (FG-NET Database)

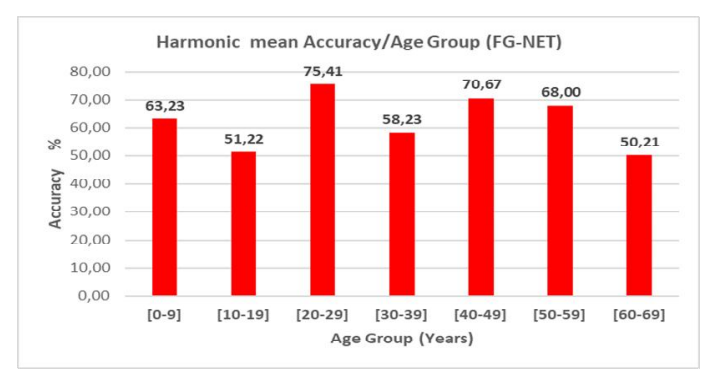

Figure 9: Performance of Harmonic mean approach vs Age Group (FG-NET Database)

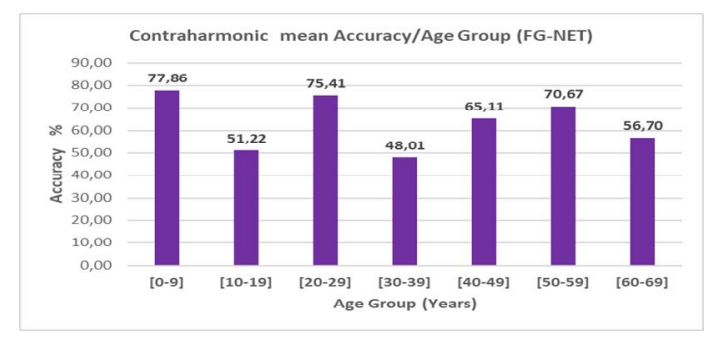

Figure 10: Performance of Contra-Harmonic mean approach vs Age Group (FG-NET Database)

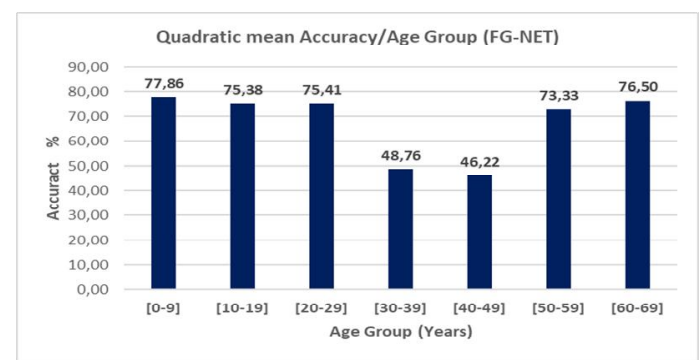

Figure 11: Performance of Quadratic mean approach vs Age Group (FG-NET Database)

The $\mathrm{x}$-axis represents the kind of the used average and the $y$-axis represents the estimation rate. The results clearly demonstrate the superiority of our algorithm to more estimate the [0-9] and the [20-29] age groups, but the last rate of estimation is that of the [30-39] age group. 
The arithmetic mean helps to classify [20-29] age group with $71.46 \%$ as estimate rate and [30-39] age group with $79.95 \%$, but it is not able to better classify the [50-59] age group. The harmonic mean helps to classify [40-49] age group with $70.67 \%$ as estimate rate, but it is not able to better classify [60-69] age group. The contra-harmonic mean helps to classify [0-9] age group with $77.86 \%$ as estimate rate, but it is not able to better classify [30-39] age group and the quadratic mean helps to classify [0-9] age group with $77.86 \%$ as estimate rate, but it is not able to better classify [40-49] age group.

The [0-9] age group is more classified by the contra-harmonic and the quadratic means. The [10-19] age group is more classified by the quadratic mean. The [20-29] group age is more classified by the arithmetic means. Also, the [30-39] group age is more classified by the arithmetic means. The [40-49] group age is more classified by the harmonic means and the both age groups [50-59] and [60-69] are more classified by the quadratic mean. the [50-59] age group. The harmonic mean helps to classify [40-49] age group with $70.67 \%$ as estimate rate, but it is not able to better classify [60-69] age group. The contra-harmonic mean helps to classify [40-49] age group with $70.67 \%$ as estimate rate, but it is not able to better classify [60-69] age group.

We tested our approach in CUHK database. Figure.13 shows the performance of the arithmetic mean algorithm per age group by years. The result tests deduct that the estimate rate reaches more than 19\% in [0-9] age group, more than $23 \%$ in [10-19] age group, more than 13\% in [30-39] age group, more than $6 \%$ in [0-9] age group and no matching score in [20-29],[40-49] and [60-69] age groups.

To use our algorithm perfectly:

- Large and labelled facial age dataset is needed with facial images useful for age estimation and classification.

- Facial frontal and net images are needed, Figure.14 shows some rejected FG-Net images by our facial estimation and classification age group (due to pose, noise...)

- A structured and multiple computing power are required for training and testing stages.

- Large memory, powerful operating system and efficient platform are demanded.

We recall that AgeGuess is an online platform which we can post our photos, other people guess our age, as well as guess the age of other users. Based on the AgeGuess idea, relatively to police investigation scenarios, based also on a query system considered as a process with the goal is to identify the corresponding image and the aim is to detect the crime and identify the suspect based on the collected information, we have tested our FAES in the case of full hidden face with a

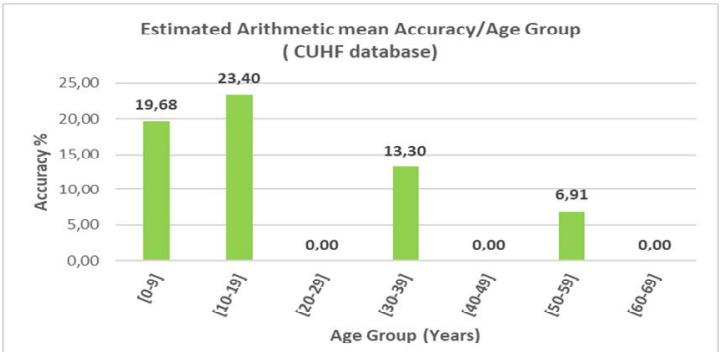

Figure 12: Performance of Arithmetic mean approach vs Age Group (CUHK Database)

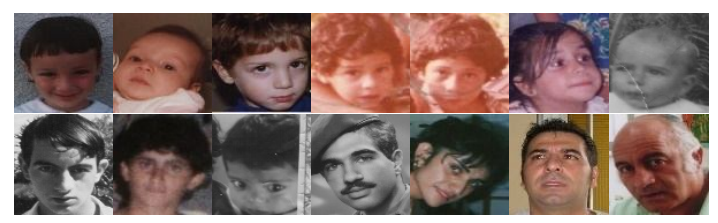

Figure 13: Some images of FG-NET Database rejected by our FAES due to pose and noise detection.

mask, case of faces from coins or banknotes, cases of statues or faces transformed to 3D images, case of stamps, case of baby doll and particularly case of human head skull wich to principal goal is a comparison of the estimated age by FAES with age result from the normal, accustomed, chemical and organic mechanisms. Figure.15 shows our returned FAES results for some random collected photos. For each image the estimated group age is: [0-9] year for Baldwin and Joker, [30-39] year for the first coin and [0-9] year for the second coin, [50-59] year for Pharaon statue, [0-9] year for Cleopatra statue,[20-29]year for Oranus statue and statue of liberty, [40-49] year for the first stump and [10-19] for the second stump, [60-69] year for the first human head skull and [10-19] for the second human head skull, [40-49]year for tow face images in the banknote and [0-9] year for the baby doll.

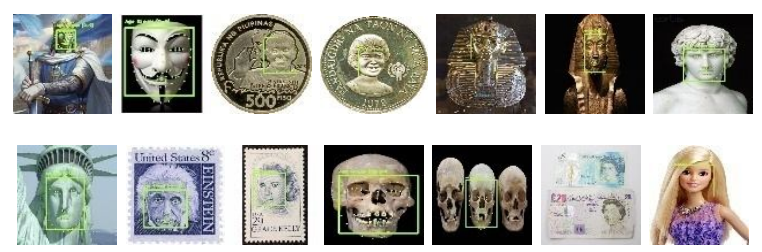

Figure 14: Results of our FAES applied to some author collected photos. (They are all slected from internet)

\section{CONCLUSION}

This paper gives a new geometrical fuzzy approach for facial age group estimation and classification. Our methods are based on Levenshtein Distance and Referential Face Ratios generated by using some useful averages. First step will be regrouping of FG-NET database images in a many small Datasubsets of age groups based on their year decade. Facial images pass though Viola -Jones facial detection algorithm. 
We extract sixteen features based on the distance between different portions of the perfect face. For each age group decades, we generate four referential faces suitable to four classical averages: Arithmetic mean, harmonic mean, contra-harmonic mean and quadratic mean. As a result of our facial estimation and classification age group system, the minimum Levenshtein distance value between the input face image request and the referential faces indexes the output probe kind of age. To study these ideas and improve our classification result, we tested our proposed methods on FG-NET and CUFS datasets. and the results is very satisfactory. Our approach Can be useful in the process of identification and retrieval of the criminal and as a future work, we plan to extend this research: we attempt to tune the same model for exact age estimation, we will add a complementary step such as rotated face images detection and integrate a comparison with face aging models.

\section{ACKNOWLEDGMENT}

I would like to express my deep gratitude to Professor A. SADIQ my research supervisor, for their patient guidance, enthusiastic encouragement and useful critiques of my research works. I would also like to thank Professors M. OUALLA \& A. SOUHAR, for their advices and assistance in keeping my progress on schedule. Authors would like to thank all the subjects that took part in the image collection for FG-NET and CUHK databases.

\section{REFERENCES}

1. P. Grd. Introduction to Human Age Estimation Using Face Images. Journal of Research papers Faculty of Materials Science and Technology Slovak University of Technology in Trnava, Special number. Vol.21, pp. 35-41. August 2013.

https://doi.org/10.2478/rput-2013-0005

2. A. Maina, B.H. Ugail and D. Connah. Automatic age and gender classification using supervised appearance model. Journal of Electronic Imaging, Vol.25. August 2016.

3. G. Xin, F.Yun and S.M. Kate. Automatic Facial Age Estimation. Tutorial at PRICAI. 2010.

4. K. Lim, N.H. Shin, Y.Y. Lee, and C.S. Kim. Order Learning and Its Application to Age Estimation. accepted to Proceedings of the International Conference on Learning Representations (ICLR). Addis Abada, Ethiopia. 2020. In press

5. K. Deepa, R.K. LekhaSree, B. Renuga Devi, V. Sadhana and S. Virgin Jenifer. Cervical Cancer Classification. International Journal of Emerging Trends in Engineering Research. Vol. 8. Pp. 804-807. March 2020. https://doi.org/10.30534/ijeter/2020/32832020

6. Aaron Don M. Africa, Anna Rovia V. Tabalan, Mharela Angela A. Tan. Speech Emotion Recognition using
Support Vector Machines. International Journal of Emerging Trends in Engineering Research. Vol. 8. Pp. 1212-1216. April 2020.

https://doi.org/10.30534/ijeter/2020/43842020

7. H. Wang, D. Huang, Y. Wang and H. Yang. Facial aging simulation via tensor completion and metric learning. IET Comput. Vis. Vol.11. pp.78-86. June 2017.

8. Y.Fu, G. Guo and T.Huang. Age synthesis and estimation via faces: a survey. IEEE Trans Pattern Anal Mach Intell. Vol.32. pp.1955-76. November 2010. https://doi.org/10.1109/TPAMI.2010.36

9. A. Lanitis, C. Draganova and C. Christodoulou. Comparing different classifiers for automatic age estimation. IEEE Trans. Man Syst. Cybern. Vol.34. pp. 621-628. February 2004

10. R. Angulu, J.R. Tapamo and A.O. Adewumi. Age estimation via face images: a survey. J Image Video Proc. 2018, Vol.42. January 2018. https://doi.org/10.1186/s13640-018-0278-6

11. K.Ounachad, A. Souhar and A. Sadiq. Fuzzy Hamming distance and Perfect Face Ratios based Face Sketch Recognition. IEEE International Congress on Information Science and Technology (CiSt) Marrakech, Morocco. pp.317-322.October 2018.

12. K.Ounachad, M. Oualla, A. Souhar and A. Sadiq. Geometric Feature Based Facial Emotion Recognition. International Journal of Advanced Trends in Computer Science and Engineering. Vol.9. pp. 3417-3425.May- June 2020. https://doi.org/10.30534/ijatcse/2020/143932020

13. Fg-net. The Fg-net Aging Database. Available from: http://www-prima.inrialpes.fr/FGnet/html/benchmarks .html. [Accessed 16th March 2020]

14. Vladimir. I. Levenshtein. Binary codes capable of correcting deletions, insertions, and reversals. Soviet Physics Doklady. Vol.10; pp707-710. Bibcode:1966SPhD...10..707L. 1966

15. J.M. Al-Tuwaijari1, M. A.B. Raheem and O.S. Salman. Intelligent System for Human Age Estimation Using Hybrid Machine Learning Technique. International Journal of Advanced Science and Technology. Vol. 29. pp. 1040-1054. January 2020.

16. W. Pei, H. Dibeklioglu, T. Baltrušaitis, and D. M. J. Tax. Attended end-to-end architecture for age estimation from facial expression videos.2019 [Online]. Available: https://arxiv.org/abs/1711.08690

17. K. Lim, N.H. Shin, Y.Y. Lee, and C.S. Kim. Order Learning and Its Application to Age Estimation, accepted to Proceedings of the International Conference on Learning Representations (ICLR). 2020. In press

18. X.Liu , Y.Zou, H.Kuang and X.Ma. Face Image Age Estimation Based on Data Augmentation and Lightweight Convolutional Neural Network. Symmetry 2020, 12, 146. 2020 https://doi.org/10.3390/sym12010146 
19. A.A Shejul, K.S. Kinage and B.E. Reddy. Facial Based Human Age Estimation Using Deep Belief Network. In: Haldorai A., Ramu A., Mohanram S., Onn C. (eds) EAI International Conference on Big Data Innovation for Sustainable Cognitive Computing. EAI/Springer Innovations in Communication and Computing. Springer, Cham DOI. https://doi.org/10.1007/978-3-030-19562-5_27.pp. 269-277. 2020

20. J.A.B. Jones, U.W.Nash, J. Vieillefont, et al. The AgeGuess database, an open online resource on chronological and perceived ages of people aged 5-100. Sci Data Vol. 6, 246 https://doi.org/10.1038/s41597-019-0245-9. October 2019

21. K.Ounachad, M. Oualla, A. Souhar and A. Sadiq. Face Sketch Recognition: Gender Classification and Recognition. International Journal of Psychosocial Rehabilitation. Vol. 24. pp.1073-1085. February 2020

22. J.Huang, Y. Shang and H. Chen. Improved Viola-Jones face detection algorithm based on HoloLens. EURASIP Journal on Image and Video Processing. Vol.12. pp.31-41. February 2019

23. M.OUALLA, K. OUNACHAD, A. HNINI and A. SADIQ. The Fast Integration of a Rotated Haar-Like Feature for Face Detection. International Journal of Advanced Trends in Computer Science and Engineering. Vol. 9.pp. 4055 - 4062. May - June 2020 https://doi.org/10.30534/ijatcse/2020/232932020

24. Y.Wu and Q. Ji. Facial Landmark Detection: A Literature Survey. International Journal of Computer Vision. Vol.127. pp.115-142 doi:10.1007/s11263-018-1097-z. February 2019. 